\title{
Spiral Coil Heat Exchanger- Experimental Study
}

\author{
Esam Jassim \\ Prince Mohammed Bin Fahd University (PMU) \\ P.O. Box 1664, Khobar, Saudi Arabia \\ ejassim@pmu.edu.sa
}

\begin{abstract}
Spiral coil offers a substantial amount of heat transfer area at a considerably low cost as it does not only have a lower wall resistance but it also achieves a better heat transfer rate in comparison to conventional U-tube arrangement. The general aim of the study is to assess different configurations of spiral coil heat exchangers that can eventually operate at a highly efficient condition. The paper documents the transient behavior of spiral-shaped tubes when the coil is embedded in a rectangular conducting slab. Different arrangements and number of turns per unit length, with fixed volumes, are considered in order to figure out the optimal configuration that maximizes the performance of the heat transfer. The implementation presented in the study is conducted to demonstrate the viability of the use of a large conducting body as supplemental heat storage. The system uses flowing water in the coil and stagnant water in the container. The copper-made coils situated in the center of the slab carries the cold fluid while the container fluid acts as a storage-medium. The water temperature at several depths of the container was measured to ensure uniformity in the temperature distribution of the container medium. Results have shown that the coil orientation, the number of loops, and the coil geometry, substantially influence the rate of the heat transfer. The outcome has concluded that the vertically-embedded spiral coil has a better performance than the horizontally-embedded spiral coil. Doubling the number of loops is shown to enhance the performance of the coil.
\end{abstract}

Keywords: Spiral Coil, Heat Exchanger, Transient Flow, Conducting Slab

\section{Introduction}

For almost a century of first introducing the curved tubes heat exchanger, much attention have recently been drawn toward the enhancement techniques in energy and mass transfer of spiral coil heat exchanger. The popularity of spiral coil heat exchanger is coming from their wide applications due to usefulness and suitability as they prove to be compact in design, unique in geometrical structures, and feasible in manufacturing. However, these virtues are not only the reasons behind the attraction of this type of heat exchanger. The eminent of such systems over straight tube type in terms of heat and mass transfer gives them domination over conventional type thermal systems. Today, with the advancements in science and technology, spiral tube heat exchangers have become prevalent in numerous thermal applications all over the world.

The design criteria of spiral tube heat exchanger vary with the requirement or their functionality [1]. For instance, in geothermal application, heat exchangers are available in two types: Open and closed type heat exchangers. The former is a direct system between the ground and the system. However, the closed heat exchanger is an indirect type of a system in which the pipes are buried in vertical or horizontal patterns. The condition of the nearby fluid determines the suitability and appropriateness of vertical versus horizontal ground heat exchangers [2].

Temperature complexities that are caused by curvature-based torsion give rise to a unique advantage to the process that can only be observed in the case of spiral pipes, [3]. Consequently, much research study and implementation of spiral pipe heat transfer exchanger are nowadays demanded for various applications.

Conventionally, spiral piping heat coupling mechanism is attributed to many factors such as geometrical configurations, compact size, bigger thermal conduction space, number of loops, etc. Numerous researches have been conducted to investigate and determine what configuration and geometry of spiral pipes could enhance the thermal coupling between them and the conducting volume. Heo et al. (2012) have described thermal coupling using spiral piping with various shapes and sizes such as length of spiral pipes, number of turns, coil radius, helix diameter etc. The natural transference of thermal energy was also studied under different conditions that include spiral pipe dimensions, rate of flow of fluid, bath temperature and inlet temperature. He proved that spiral shape is more effective than U-shape in terms of rate of heat transfer, [4]. 
Other studies conducted by different researchers have emphasized the fact that spiral tubes are indeed better than any other form of tubes and effectively act as heat transfer enhancers. Therefore, despite the differences of studies on spiral pipe usage in industrial application, the outcome results of both experimental and analytical studies are end up to same conclusion.

Spiral coil buried in a conducting volume has been scarcely addressed in the literatures. Applications of such type heat exchanger include geothermal heat pumps, extracting oil and gas, and other solid-fluid interaction applications. Despite several numerical, analytical, and experimental papers have been published to address the heat transfer mode of embedded coil, limited studies have been conducted to optimize the configuration and orientation of the coil as well as the heat transfer mechanism during transient process. Parameters such as number of loops per unit tube length, mass flow rate, coil orientation, and coil pitch are some of many factors that influence the heat transfer efficiency and yet their effects on coil performance and heat rate are needed to be further emphasized. The main objectives of this paper include studying and analysing experimentally the behaviour of spiral coil embedded in a conducting container, highlighting the impact of orientation and number of loops on the coil performance, and presenting the spatial and temporal mechanisms of the heat transfer process

\section{The Transient Surface Temperature}

Consider a conducting volume contains a medium (water) of mass $M$, volume $V$, density $\rho$, and specific heat $C_{p}$ initially at a uniform temperature $T_{w i}$. At time $t=0$, a coiled-tube pipe with surface area $A_{s}$, carrying fluid of mass flow $\dot{m}_{l}$, is sunk into the medium. Energy transfer occurs between the medium in the container and the fluid inside the coil by natural convection $(h)$. For simplicity, we assume that transient temperature of the medium $T(\mathrm{t})$ is less than its initial temperature $T_{w i}$, however, the energy analysis could also be used for the opposite case. We also assume that the medium inside the container has uniform instantaneous temperature however, it changes only with time, $T=T(t)$. The analysis ignores the coil thermal resistance since it is made of high conductive material (copper).

The temperature of the medium drops by a differential value $d T$ for a differential time interval $d t$. The energy balance of the system for the time interval $d t$ can be expressed as:

$$
\begin{gathered}
M \cdot C_{p} \cdot \frac{d T}{d t}=-h \cdot A_{s} \cdot\left(T-T_{s}\right) \\
\left(\frac{T(t)-T_{s}}{T_{w i}-T_{s}}\right)=\boldsymbol{e}^{-\boldsymbol{B} \cdot t}
\end{gathered}
$$

where

$$
B=\frac{h \cdot A_{s}}{\rho V C_{p}}
$$

The surface area of the coil will be compared for two cases. The first case is ignored the effect of the curvature and assume the 1-D conventional tube surface area is applied; i.e. $\left(A_{\mathrm{s}}=\pi d_{p} l\right)$ where $l$ is the coil length. In contracts, the second case considers the coil geometry by introducing the total outside heat transfer area $\left(A_{\text {coil }}\right)$ for spiral coil. The area can be obtained from the following expression, [5]:

$$
A_{\text {coil }}=\pi \cdot D \cdot A_{o} \cdot n(1+2 \cdot \pi \cdot \alpha \cdot n / D)
$$

where $A_{o}$ stands for the heat transfer area per unit length $\left(\pi d_{p}\right) ; n$ is number of turns; $D$ is helix diameter; and $\alpha$ is the rate of increase of helix radius, in ( $\mathrm{m} / \mathrm{rad})$.

The external natural convective heat transfer coefficient $(h)$ for each time step can be obtained from the following expressions [6]:

For Vertical Positioned Coil: 


$$
\mathrm{Nu}=\frac{h \cdot H_{t}}{k}=\left\{0.825+\frac{0.387 \cdot R a_{L}^{1 / 6}}{\left[1+(0.492 / P r)^{9 / 16}\right]^{8 / 27}}\right\}^{2}
$$

While for horizontal positioned coil:

$$
\mathrm{Nu}=\frac{h \cdot D}{k}=\left\{0.6+\frac{0.387 \cdot R a_{L}^{1 / 6}}{\left[1+(0.599 / P r)^{9 / 16}\right]^{8 / 27}}\right\}^{2}
$$

$H_{t}$ is the height of the coil, $D$ is the helix diameter (Fig. 1-a), and $R a$ is the Rayleigh number.

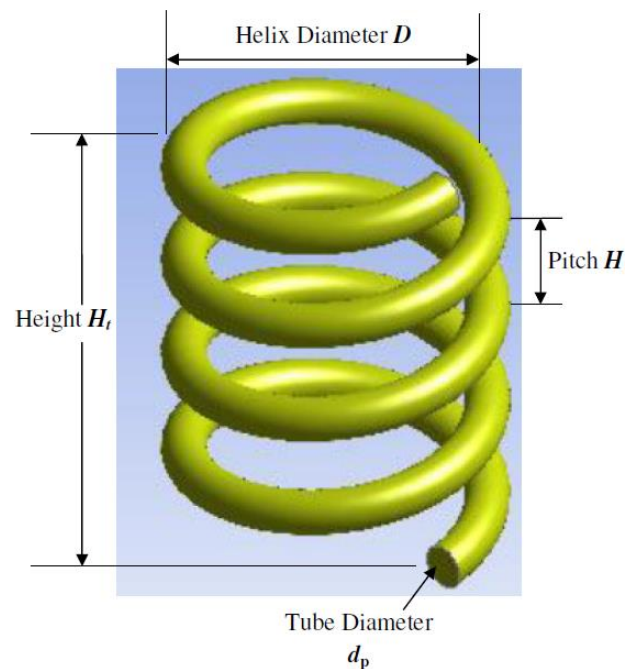

(a)

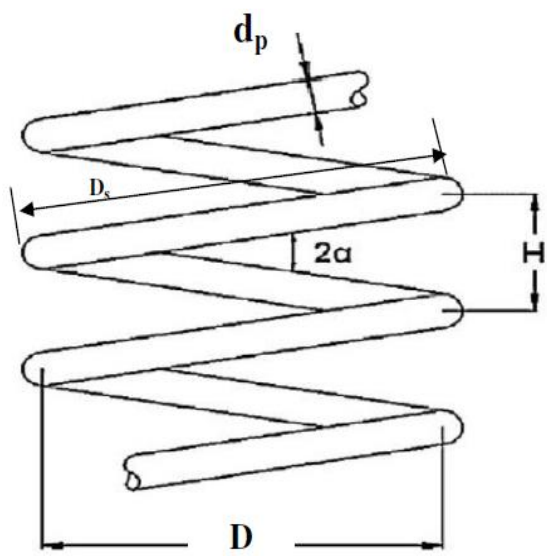

(b)

Fig. 1: Typical geometry of Spiral Coil $(a)$ isometric; $(b)$ Plane view.

Helix diameter is obtained from the following expression [7]:

$$
D=\frac{\sum_{i=1}^{N} \sqrt{D_{s i}^{2}-\left(\frac{H_{t}}{\pi}\right)^{2}}}{N}
$$

where $\boldsymbol{D}_{\boldsymbol{s i}}$ stands for the slanted outer turn diameter for each loop (Fig.1-b).

\section{Experimental Set-up}

For the purpose of the experiments, a $19 \mathrm{~mm}$ diameter copper-tubes coils each of $10.6 \mathrm{~m}$ length are used. Only $5 \%$ of the length is straight before and after the helical portion. The tubes are carefully furnished from inner surface to reduce the flow resistance. External pipes are connected to the coil with straight joints at the two ends. Insulation was applied to the external pipes to avoid any heat gain/loss from the surroundings other than the container. Thermal insulation material is covering the conducting volume (container) to minimize the heat losses to the surroundings.

Four electric heaters with a capacity of $2500 \mathrm{~W}$ each are mounted at the bottom of the container to provide heat energy to the water of the container. Thermal regulator is installed to stabilize the water pool temperature within $\pm 0.1^{\circ} \mathrm{C}$ precision. The pump and the ball valve regulate the flow of fluids inside the spiral pipes. The ball valve is specifically designed to maintain a particular flow rate of fluid flowing within the coil when the pump is under operation. A centrifugal pump is connected to push the fluid through the spiral pipes at varying Reynolds numbers. 
During the experiment, the fluid flowing inside the coil absorbs heat energy from the water of the container while passing through the spiral tube. The inlet/outlet temperatures of the spiral pipes are measured using the installed digital thermometers. Similarly, the inlet/outlet pressures were measured too using the installed pressure transducers. Important data were captured momently and recorded until steady state occurs.

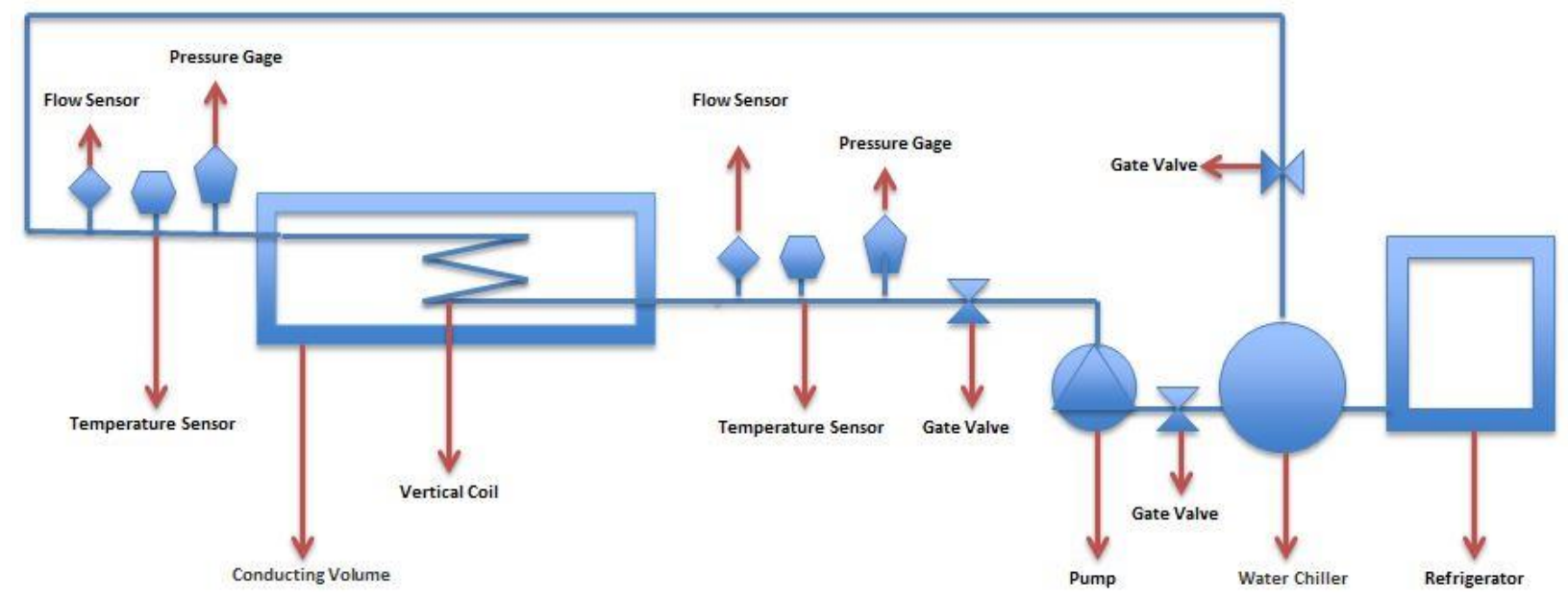

Fig. 2: Experimental Apparatus.

\section{Results and Discussion}

Experiments are carried out for different boundary conditions and coil parameters such as: coil pitch, number of loops, Reynolds number, and coil orientation. The constraints of the experiments are illustrated in table (1).

Table 1. Thermal Properties and System Dimensions.

\begin{tabular}{|c|c|c|}
\hline Property & Unit & Value \\
\hline Pipe diameter & $\mathrm{mm}$ & 19 \\
Coil Length & $\mathrm{m}$ & 10.6 \\
Container $(l \times w \times \mathrm{h})$ & $\mathrm{m}$ & $1.14 \times 0.65 \times 0.48$ \\
density & $\mathrm{kg} \cdot \mathrm{m}^{-3}$ & 994 \\
Dynamic viscosity & $\mathrm{kg} \cdot \mathrm{m}^{-1} \cdot \mathrm{s}^{-1}$ & $0.653 \times 10^{-3}$ \\
Thermal conductivity & $\mathrm{W} \cdot \mathrm{m}^{-1} \cdot \mathrm{K}^{-1}$ & 0.648 \\
Specific heat & $\mathrm{J} \cdot \mathrm{kg}^{-1} \cdot \mathrm{K}^{-1}$ & 4190 \\
Prandtl Number & & 4.23 \\
\hline
\end{tabular}

\subsection{Number of Loops}

The coil selected for studying the influence of its geometry has helix diameter of $(\mathrm{D}=15 \mathrm{~cm})$, Pitch of $(\mathrm{H}=2 \mathrm{~cm})$, and 10 loops. The coil was firmly mounted vertically and sunk completely inside the pool. Coil inlet/exit temperatures and pressures, container medium temperature, and flow rate have been recorded each minute.

Another coil of 5 loops with same length and pitch but different loop diameter $(30 \mathrm{~cm})$ is vertically investigated under similar conditions illustrated in table 1 . The behaviour of the coil due to the change in the number of loops are presented in Figs.(3-5). The comparison of the normalized temperature of the container medium is clearly shown that increasing the number of loops would increase the rate of heat transfer despite the identical length of the two coils.

Figure (4) proved that disregarding the curvature of the coil, when theoretical design is conducted, would lead to insignificance impact of the loops on the coil performance. Hence, one would expect remarkable erroneous in the designed parameters during the process of analysis of the spiral coil heat exchanger.

Coil efficiency of course is expected to be improved following the enhancement occurred in the rate of the heat transfer as depicted in Fig. (5). 


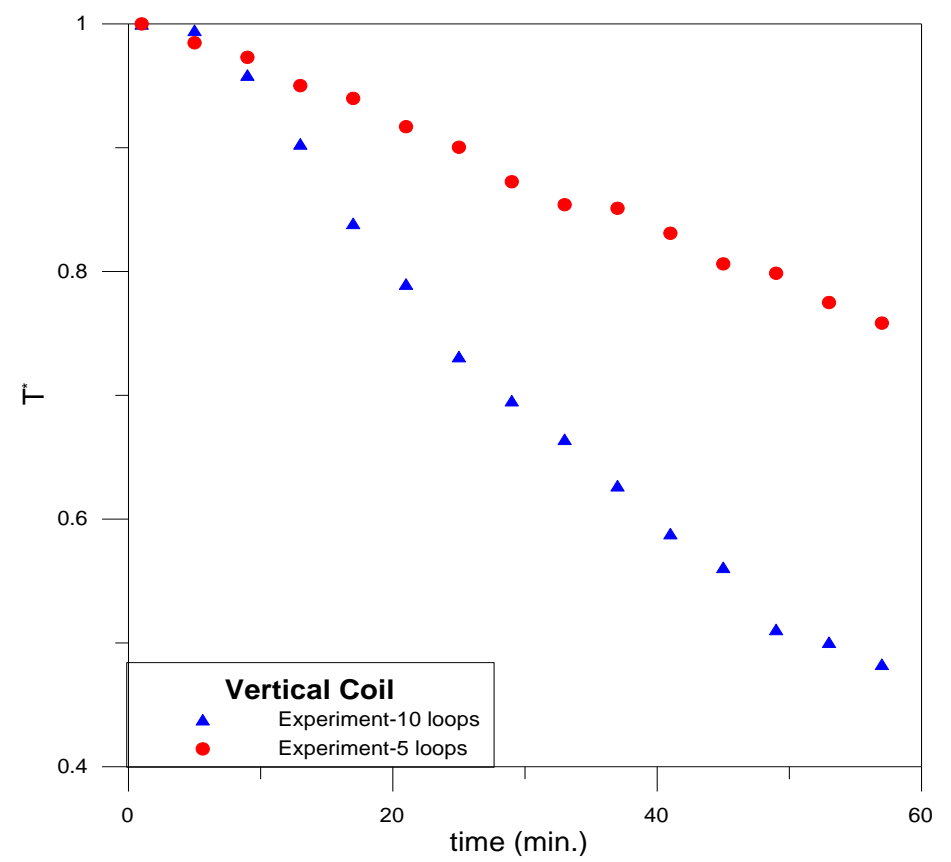

Fig. 3: Influence of Number of loops.

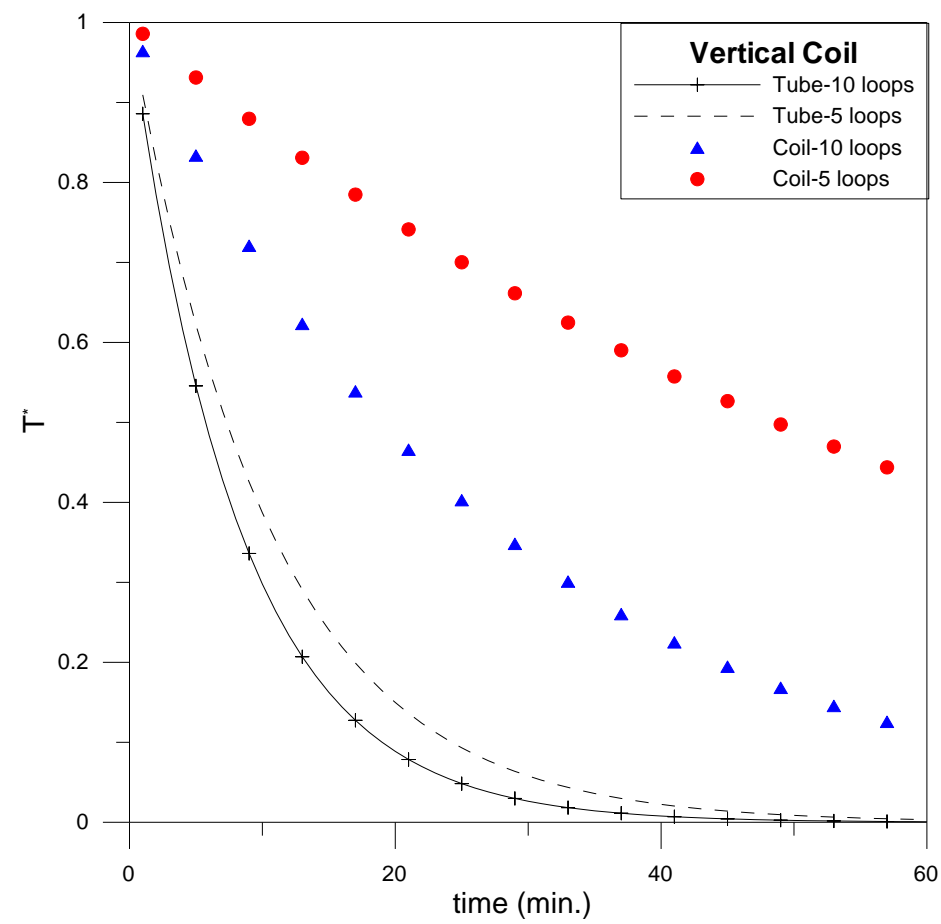

Fig. 4: Comparison of 1-D analysis for straight tube vs. coil. 


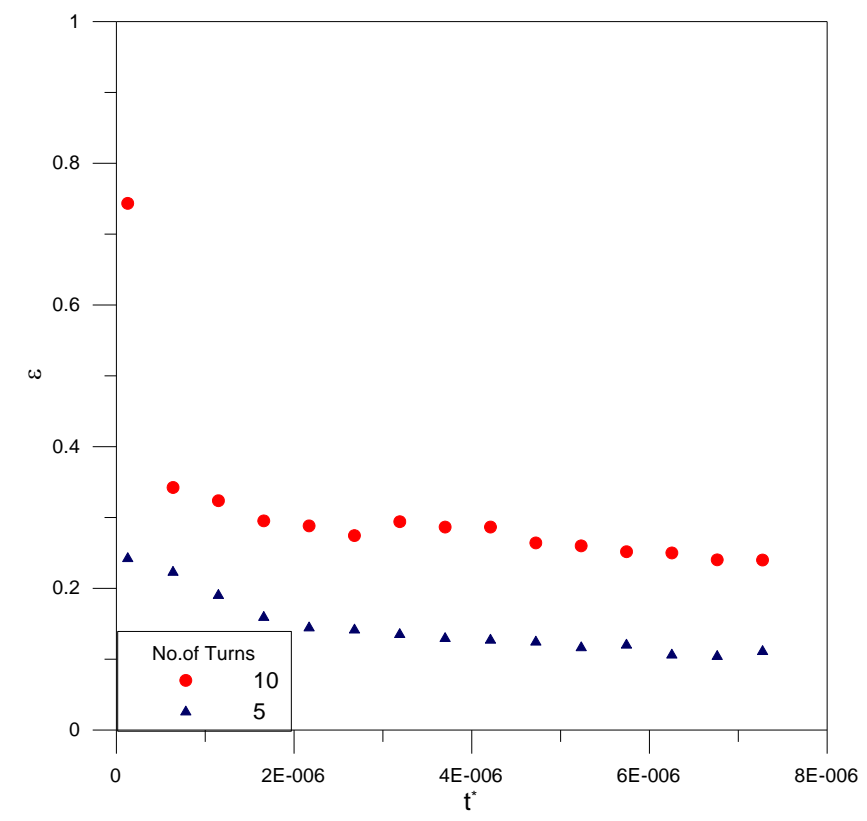

Fig. 5: Effect of coil loops on the performance.

\subsection{Coil Orientation}

To investigate the impact of orientation, the 10 loops vertical coil is fabricated to sink horizontally in the container. Fig. (6) presents the normalized temperature variation with time step for vertical and horizontal positions. Based on the experiment results, fixing the coil vertically is shown to be better than the horizontal position in terms of transferring heat to the container's fluid. However, no such outcome is obtained when 1-D analysis is applied even though the coil curvature is considered. This important conclusion manifests the drawback of using the 1-D analysis of the coil and sustains the demand of experimental studies for obtaining reliable results.

The performance of the vertically-positioned coil is shown to be slightly enhancer than horizontal position as presented by Fig. 7.

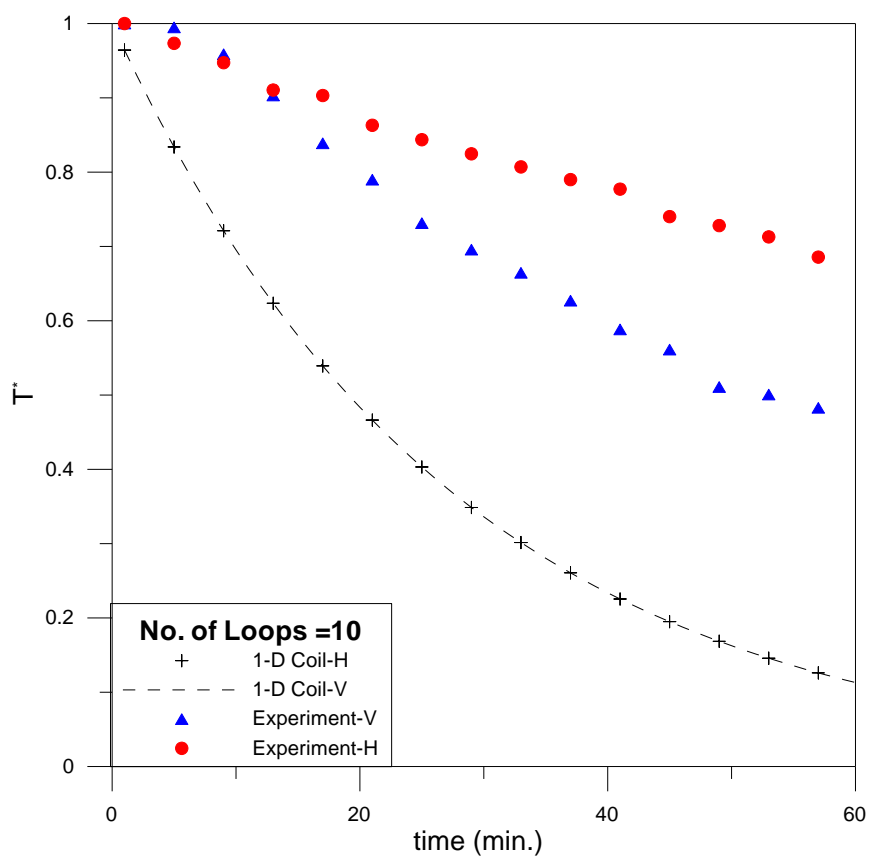

Fig. 6: Temperature variation predicted by 1-D coiled-type and experiment. 


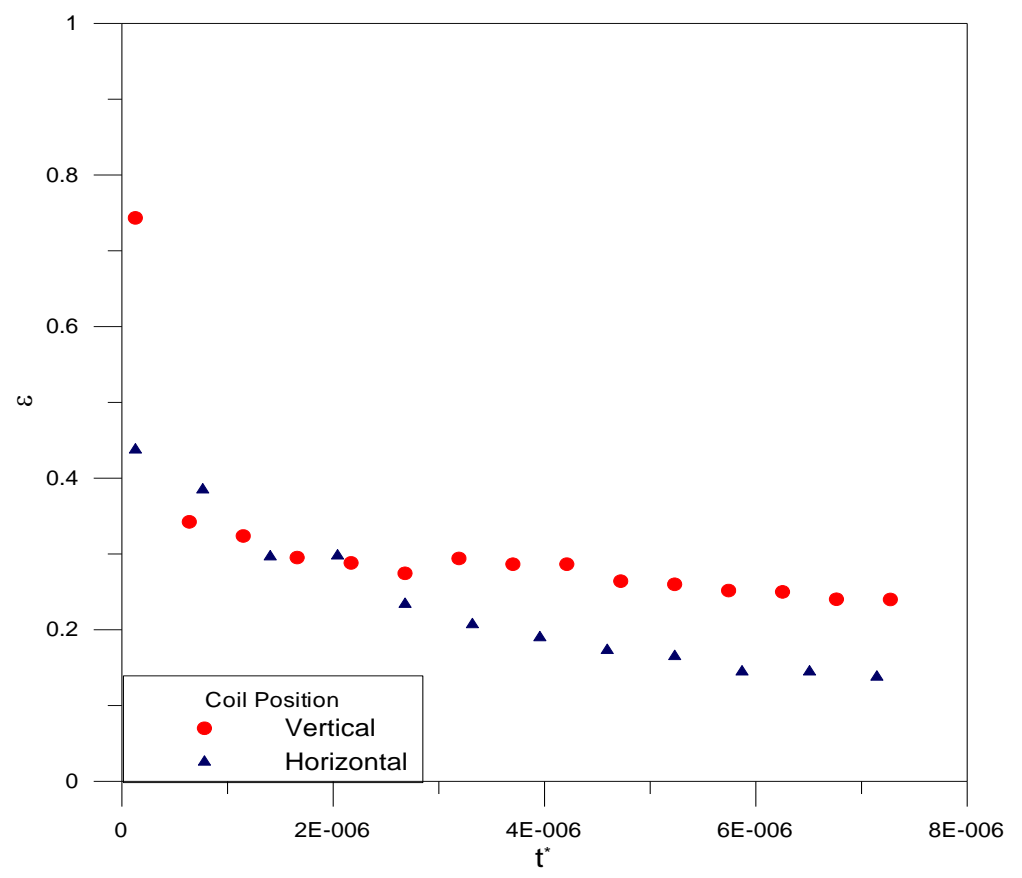

Fig. 7: Comparison of efficiency between vertical vs. horizontal coil.

\section{Conclusion}

Experimental studies on transient natural convection heat transfer from spiral coil are conducted. Impact of coil geometry, orientation, and number of loops are documented. The following summarize the outcomes:

1. Vertically-positioned coil was shown to be more effective than horizontal coil in terms of transferring heat.

2. Although including the coil curvature during the theoretical analysis readably interprets the actual behaviour of the coil, it was shown that such analysis has failed to predict the effect of coil orientation. This would support the demand of experimental investigation as an alternative robust method.

3. Increasing number of loops per unit length of spiral coil enhances the rate of heat transfer.

4. Uncertainty analysis showed that the accuracy of the results is within $\pm 5 \%$.

\section{Acknowledgements}

The author would like to express his gratitude to the Prince Mohammed University (PMU) for supporting and sponsoring this work.

\section{References}

[1] B. Sanner, C. Karytsas, D. Mendrinos, and L. Rybach, "Current status of ground source heat pumps and underground thermal energy storage in Europe," Geothermics., vol. 32, no. 4, pp. 579-588, 2003.

[2] M. Alalaimi, S. Lorente, and A. Bejan, "Thermal coupling between a spiral pipe and a conducting volume," International Journal of Heat and Mass Transfer, vol. 77, pp. 202-207, 2014.

[3] A. Sasmito, J. Kurnia, and A. Mujumdar, "Numerical evaluation of laminar heat transfer enhancement in nanofluid flow in coiled square tubes," Nanoscale research letters, vol. 6, no. 1, pp. 1-14, 2011.

[4] J. Heo and B. Chung. "Influence of helical tube dimensions on open channel natural convection heat transfer," International Journal of Heat and Mass Transfer, vol. 55, no. 11, pp. 2829-2834, 2012.

[5] N. Wijeysundera, J. Ho, and S. Rajasekar, "The Effectivness of a Spiral Coil Heat Exchanger," Int. Comm. Heat and Mass Transfer, vol. 23, no. 5, pp. 623-631, 1996.

[6] A. Yunus and J. Afshin, Heat and Mass Transfer, $4^{\text {th }}$ Edition. MeGraw Hill, 2010.

[7] M. Ali, "Natural convection heat transfer from vertical helical coils in oil," Heat Transfer Engineering, vol. 27, no. 3, pp. 79-85, 2006. 